\title{
A Realistic Model of the Inner Organs from the Visible Human Data
}

\author{
K. H. Höhne ${ }^{1}$, B. Pflesser ${ }^{1}$, A. Pommert ${ }^{1}$, M. Riemer ${ }^{1}$, R. Schubert ${ }^{1}$, T. Schiemann ${ }^{1}$, \\ U. Tiede ${ }^{1}$, U. Schumacher ${ }^{2}$ \\ ${ }^{1}$ Institute of Mathematics and Computer Science in Medicine, \\ ${ }^{2}$ Institute of Anatomy \\ University Hospital Eppendorf, \\ D-20246 Hamburg, GermanyHamburg, Germany \\ \{hoehne, pflesser, pommert, riemer, schubert, tiede, \\ schumacher\}@uke.uni-hamburg.de
}

\begin{abstract}
The computer-based 3D models of the human body reported to date suffer from poor spatial resolution. The Visible Human project has delivered high resolution cross-sectional images that are suited for generation of highquality models. Yet none of the $3 \mathrm{D}$ models described to date reflect the quality of the original images. We present a method of segmentation and visualization which provides a new quality of realism and detail. Using the example of a 3D model of the inner organs, we demonstrate that such models, especially when combined with a knowledge base, open new possibilities for scientific, educational, and clinical work
\end{abstract}

\section{Introduction}

While in classical medicine, knowledge about the human body is represented in books and atlases, present-day computer science allows for new, more powerful and versatile computer-based representations of knowledge. Their most simple manifestations are multimedia CD-ROMs containing collections of classical pictures and text, which may be browsed arbitrarily or according to various criteria. Although computerized, such media still follow the old paradigm of text printed on pages accompanied by pictures. This genre includes impressive atlases of cross-sectional anatomy, notably from the photographic cross-sections of the Visible Human Project (1-3). In the past years, however, it has been shown that pictorial knowledge, especially about the structure of the human body, may be much more efficiently represented by computerized $3 D$ models (4), which can be constructed from crosssectional images generated by computer tomography (CT), magnetic resonance imaging (MRI), or histologic cryosectioning, as in the case of the Visible Human Project. Such models may be used interactively on a computer screen or even in "virtual reality" environments. If such models are connected to a data base of descriptive information, they can even be interrogated or disassembled by addressing names of organs (4-6). They can thus be regarded as a "self-explaining body". 
Until now, the Visible Human Project has not reported 3D models that reflect the rich anatomical detail of the original cross-sectional images. This is due to the fact that, for the majority of anatomical objects contained in the data, the cross-sectional images could not be converted into a set of coherent realistic surfaces. If, however, we succeed in converting all the detail into a 3D model, we gain an unsurpassed representation of human structure that opens new possibilities for learning anatomy and simulating interventions or radiological examinations. This paper presents a new technique for segmenting and modeling the 3D anatomy of the Visible Human data that leads to a nearly photorealistic computer based model.

\section{Earlier Work}

Work on 3D anatomical reconstruction from spatial sequences of cross-sectional medical images goes back to the late seventies (7). Since then, techniques have been improved continuously (8-10). However, the impact of these techniques on practical medicine remains limited, because the procedure for creation of $3 \mathrm{D}$ models is rather complex. The process of $3 \mathrm{D}$ visualization requires - in contrast to classical 2D imaging - a processing step that identifies the regions in the data volume that correspond to anatomical objects. Otherwise we could not unveil their surfaces or remove objects obscuring the ones behind. This step is called segmentation. A common approach to $3 \mathrm{D}$ visualization which tries to avoid this step is volume rendering. Instead of an explicit identification of organs, transparency values are assigned to the image volume according to the color values or color changes at the object borders. In the case of the Visible Human data, this method (11) yields semitransparent views with acceptable quality e. g. for the outer surface, the muscles and the skeleton. It fails, however, to display internal structures properly. In addition, organ borders are not explicitly indicated, thus making the removal or exclusive display of an organ impossible.

Segmentation, i. e. the exact determination of the surface location of an organ is crucial to its realistic display. Complete automatic segmentation using methods of computer vision fails, since the capabilities of computers are far inferior to those of a human observer. The brute force approach to segmentation is manual outlining of objects on the cross-sections (11). Besides the fact that this procedure is tedious and very time consuming, it is largely observer-dependent and thus does not yield exact and coherent surfaces. Furthermore, despite the high resolution of the data set, important details such as nerves and small blood vessels cannot clearly be identified, because their size and contrast is too small even at the high resolution of the Visible Human data set.

\section{Material and Methods}

We therefore aimed at a method that yields surfaces for the segmentable organs that are as exact as possible and textured with their original color. In order to arrive at a 
complete model, we decided to model non segmentable objects like nerves and small blood vessels artificially on the basis of landmarks present in the image volume.

Data. Photographic cross-sectional images from the male Visible Human were used to create models of the head, neck, and torso as well as the corresponding inner organs. The original data set consists of 1871 photographic cross-sections with a slice distance of $1 \mathrm{~mm}$. The cross-sections themselves have a spatial resolution of $1 / 3 \mathrm{~mm}$. For the model of the inner organs, the resolution was reduced to $1 \mathrm{~mm}$ for reasons of data storage and computing capacity. From 1049 such slices, an image volume of $573 \times 330 \times 1049$ volume elements ("voxels") of $1 \mathrm{~mm}^{3}$ was composed, where each voxel is represented by a set of red, green and blue intensities ("RGB-tuple"). The Visible Human data set also includes two sets of computer tomographic images of $1 \mathrm{~mm}$ slice distance, one taken from the fresh, the other (like the photographic one) from the frozen cadaver. Both were transformed into an image volume congruent with the photographic one.

Segmentation. The image volume thus created was segmented with an interactive tool, which is an extension of an earlier development for RGB data (13). It can be regarded as an "electronic sculpturing tool". On a cross-section, an expert "paints" a typical region of the organ under consideration. All voxels in the volume with similar RGB-tuples are then collected by the program to form a "mask". This mask usually needs to be refined by repeating this procedure in order include the target organ completely. The resulting cluster in RGB space has an ellipsoid shape, due to the slight correlation of the colors; this cluster is then converted into the mathematical form of an ellipsoid, which facilitates subsequent computations. There are, of course, other regions with the same characterization present in the volume. If they are not connected to the target organ, it can be isolated easily; if not, 3D interactive cutting tools are used.

Graphic modeling. There are many anatomical constituents, such as nerves and small vessels, which are too small to be segmented adequately, but need to be included in a comprehensive anatomical model. For these, we have developed a modeling tool that allows us to include tube-like structures into the model. Ball-shaped markers of variable diameter are imposed by an expert onto the landmarks still visible on the cross-sections or on the 3D image; these markers are automatically connected to form tubes of varying diameter. Unlike the segmented objects, which are represented as sets of voxels, the modeled structures are represented as composed of small triangles.

Knowledge modeling. Each voxel of the spatial representation of anatomical data thus gained is connected to a knowledge base containing object descriptions within the structure of a semantic network $(4,14,15)$. Different networks were created for different views (e. g. systematic or topographic anatomy). Within the views, the anatomical constituents are linked by relations like part of, has part, branching from or branching to. 
Visualization. The 3D visualization algorithm we have developed is characterized by the fact that it renders surfaces from volume data. This is different from the volume rendering typically used for visualizing the Visible Human data set. As in volume rendering, the program casts rays from an image plane onto the image volume in viewing direction. However, the rays stop at the first encountered object. Surface texture and surface inclination (important for proper computation of light reflection) are calculated from the RGB-tuples at the segmented border line. The decisive quality improvement is achieved by determining the object surfaces with a spatial resolution higher than the resolution of the original voxels. While such subvoxel resolution may be achieved easily by sampling with high rates in volume rendering, the determination of distinct adjacent surfaces in subvoxel resolution is a major problem that has been solved only recently (16). This makes the high resolution visualization of distinct surfaces possible, which is the characteristic feature of our approach.

The objects modeled with the surface modeler are visualized with standard computer graphics methods within the context of the volume objects. The visualization program, an extended version of the VOXEL-MAN system (4), runs on UNIX workstations. Because of the high resolution and the sophisticated algorithms, the computation of a single image may take several minutes, even on a high end workstation. The model is therefore being made available for interaction and exploration via precomputed "Intelligent QuickTime VR Movies" (17), which may be viewed on any personal computer.

\section{Results}

Using the methods described above, we have built a model of the organ systems within the torso of the male Visible Human. It contains more then 850 threedimensional anatomical constituents (not just labeled cross-sections) and more than 2000 relations between them.

The following features of this model represent innovations:

- Because of the exact segmentation and the visualization method developed, the visual impression is one of unsurpassed realism;

- There is, to date, no computer model of human structure that contains so many three-dimensional organs;

- The model is space-filling i. e. any voxel is labeled as an element of a 3D object;

- The integrated formal organization of pictorial and descriptive information allows a virtually unlimited number of ways of using the model.

The model is a general-knowledge representation of gross anatomy, from which all classical representations (pictures, movies, solid models) may be derived via mouse click. In addition, the model may be used interactively for teaching and simulation of diagnostic and therapeutic procedures. While the underlying principle was reported earlier (4), the model we describe is the first to offer sufficient detail and comprehensiveness to serve these purposes seriously. FIG. 1 gives an impression of image quality and the degree of detail. 


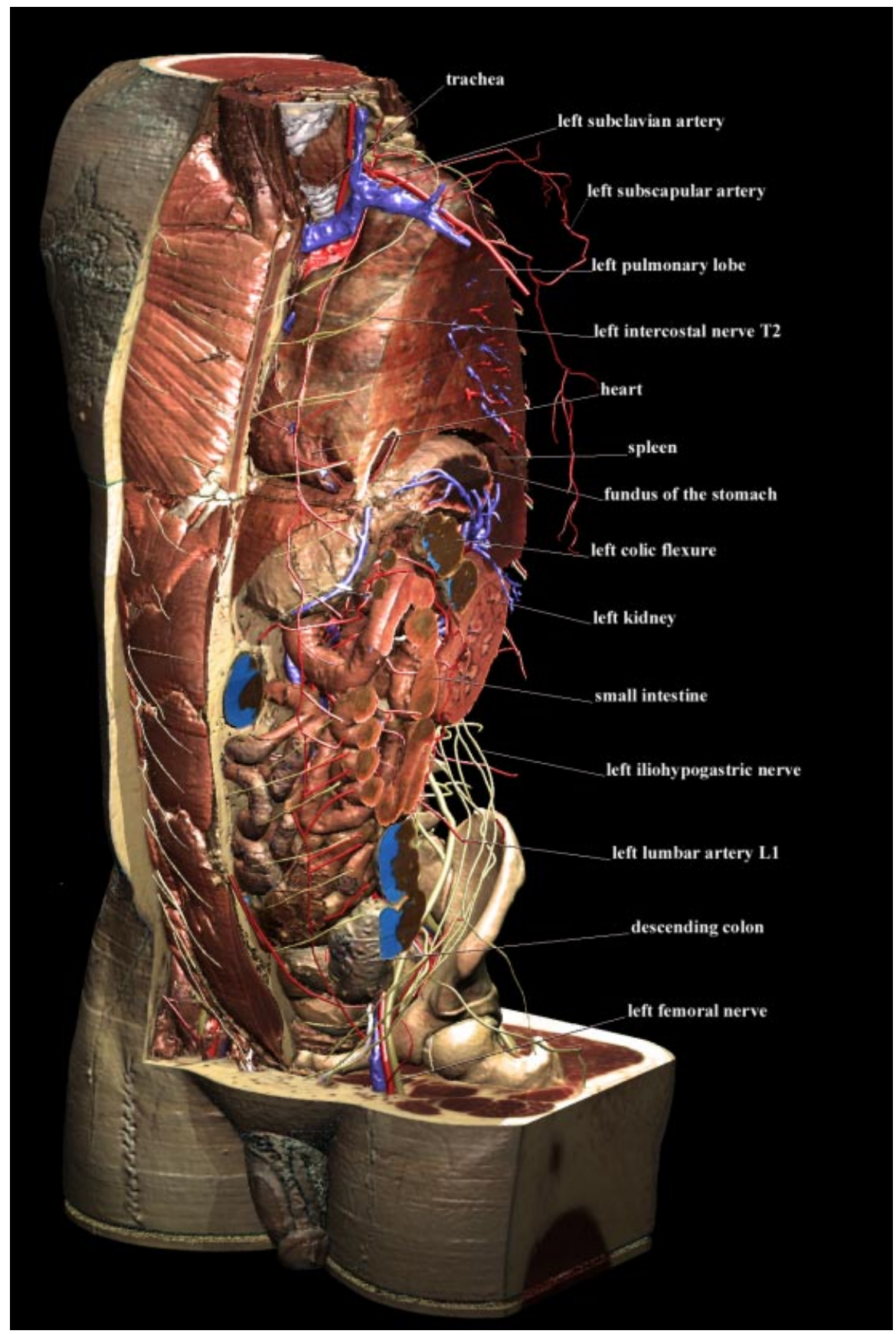

Fig. 1. The model can be viewed from any direction, cuts may be placed in any number and direction and objects may be removed or added. Annotations may be called by mouse click. 


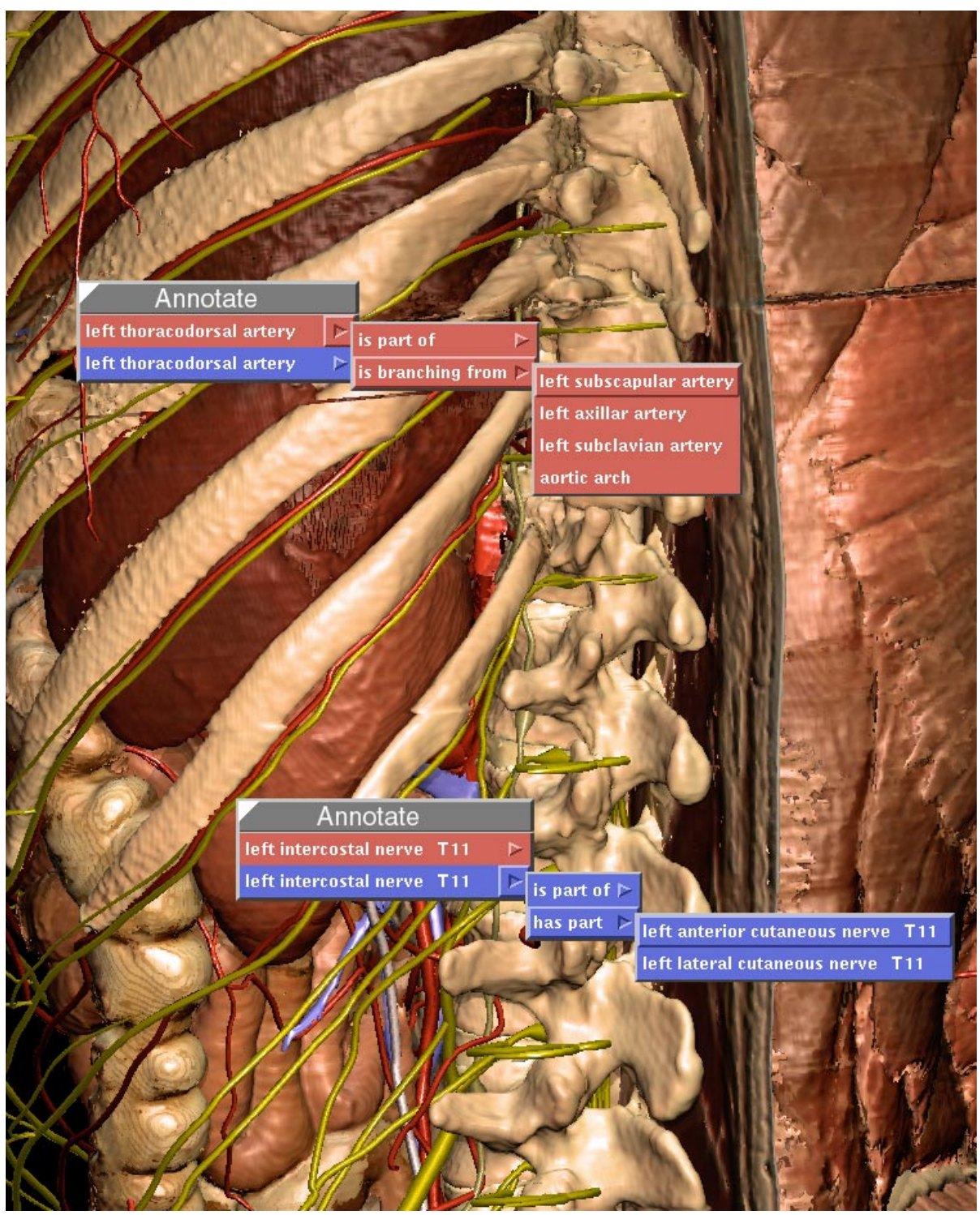

Fig. 2. Inquiring the semantic network behind the pictorial model according to different "views". The user has clicked onto a blood vessel and a nerve and received information about systematic(red) and topographic anatomy(blue). 


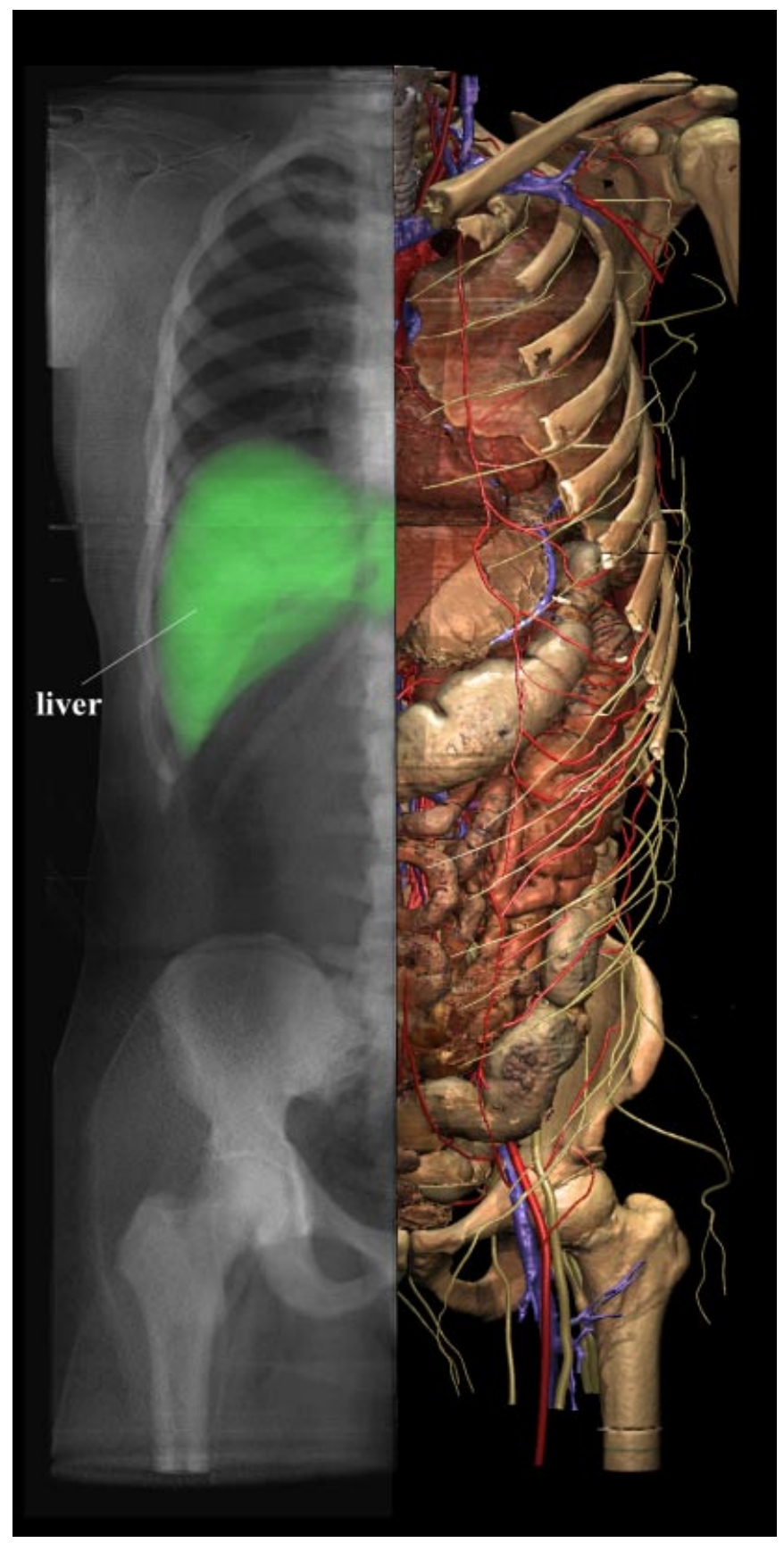

Fig. 3. Different viewing modes such as X-ray imaging may be chosen from any direction and for any part of the image. The X-ray image may be interrogated as to which object contributes to the image. 
Since the model is volume-based, cut planes (which can be placed in any number and direction) show the texture of the original cross-sectional images and thus look realistic (FIG. 1). This virtual dissection capability not only allows an interactive dissection for learning purposes, but also simulates the rehearsal of a surgical procedure. In addition, the image of a "self-explaining body" allows us to inquire about complex anatomical facts. FIGures 1 and 2 show different possibilities for presenting these explanations. The more traditional way of annotating structures of interest is demonstrated within the user-specified 3D scene in FIG. 1. These annotations can be obtained simply by pointing and clicking with the mouse on the structure of interest. However, pressing another button of the mouse will result in popup menus shown in FIG. 2; these offer structured knowledge about anatomy and function. Such information is available because every voxel, and therefore any visible point of any user-created 3D-scene, is linked to the knowledge base.

A special feature of the model involves the possibility of simulating radiological examinations (FIG. 3). Since the absorption values for every voxel are available in the original tomographic data, artificial X-ray images from any direction can be computed. Based on the information of the model, both the contributing anatomical structures and the extent of their contribution to the final absorption can be calculated. Similarly, the information present in cross-sectional radiological images (Computer Tomography, Magnetic Resonance Imaging, Ultrasound) can be clarified by presenting them in the corresponding $3 \mathrm{D}$ context.

\section{Discussion}

Computer-based models of the human anatomy created to date are more like sophisticated video games than they are professional tools for medical education and practice. We have presented a general approach for creating models that have the spatial resolution, the degree of detail, and the organization suitable for useful application in educational, scientific, and clinical work. This has been demonstrated with the example of a 3D model of the inner organs derived from the Visible Human data set. The versatility of the approach makes it suitable for anatomy and radiology teaching as well as for simulation of interventional procedures. Last, but not least, it can be used to provide patients with necessary information. A first version of a 3D anatomy atlas based on the described concept has been published recently (18).

Yet there are still improvements to be made. Like all postmortal images, those of the Visible Human suffer from postmortal artifacts, such as collapsed blood vessels. These artifacts cannot be corrected completely. One more severe limitation is the fact that the data is derived from one single individual. The interindividual variability of organ shape and topology in space and time is thus not yet part of the model. Inclusion of variability into 3D models is a difficult problem not yet generally solved. So far most progress has been achieved for 3D atlases of the brain (19).

However, the current model should be an excellent basis for further developments. One such development is the inclusion of physiology, e. g. the modeling of blood flow or propagation of electrical fields throughout the body. Applications such as the 
computation of body surface potential maps (20) should profit from an increased level of detail. Furthermore, because of the more detailed characterization of tissues, surgical simulation involving soft tissue deformation could be made much more realistic (20). This approach is thus an important, albeit early step towards computer models that not only look real, but also act like a real body.

\section{References}

1. Spitzer, V., Ackerman, M. J., Scherzinger, A.L., Whitlock, D.: The visible human male: a technical report. J-Am-Med-Inform-Assoc 1996; 3(2):118-30.

2. Ackerman MJ. The Visible Human Project: a resource for anatomical visualization. Medinfo. 1998; 9 Pt 2:1030-2.

3. Spitzer VM, Whitlock DG. The Visible Human Data Set: the anatomical platform for human simulation. Anat-Rec. 1998 Apr; 253(2):49-57.

4. Höhne, K. H., Pflesser, B., Pommert, A., Riemer, M., Schiemann, T., Schubert, R., Tiede, U. A new representation of knowledge concerning human anatomy and function. Nature Med. 1995; 1(6):506-511.

5. Rosse C, Mejino J, Modayur B, Jakobovits R, Hinshaw K, Brinkley JF. Motivation and organizational principles for anatomical knowledge representation: the digital anatomist Symbolic Knowledge Base. J-Am-Med-Inform-Assoc 1998; 5(1): 17-40.

6. Golland P, Kikinis R, Halle M, Umans BA, Grimson WEL, Shenton ME et al. AnatomyBrowser, A novel approach to visualisation and integration of medical information. Computer Assisted Surgery 1999; 4: 129-143.

7. Herman GT, Liu HK. Display of three-dimensional information in computed tomography. Journal of Computer Assisted Tomography 1977;1:155-160.

8. Höhne, K. H., Bernstein R. Shading 3D-images from CT using gray level gradients", IEEE Trans. Med. Imaging 1986; 5(1):45-47.

9. Levoy M. Display of surfaces from volume data. IEEE Comput Graphics and Appl.1988; $8(3): 29-37$

10. Tiede, U., Bomans, M., Höhne, K. H., Pommert, A., Riemer, M., Wiebecke,G.: Investigation of medical 3D rendering algorithms. IEEE Comput. Graphics. Appl. 1990;10(2):41-53.

11. Stewart, J. E., Broaddus, W.C., Johnson, J.H.: Rebuilding the Visible Man. In: Höhne, K. H. and Kikinis R, editors. VBC 96. Proceedings of the Conference on Visualization in Biomedical Computing; 1996 Sep 22-25; Hamburg, Germany. Berlin Heidelberg: Springer-Verlag 1996. Lecture Notes in Computer Science 1131, p. 81-85

12. Mullick, R., Nguyen, H.T.: Visualization and Labeling of the Visible Data Set: Challenges and Resolves. In: K. H. Höhne and R. Kikinis, editors. VBC 96. Proceedings of the Conference on Visualization in Biomedical Computing; 1996 Sep 22-25; Hamburg, Germany. Berlin Heidelberg: Springer-Verlag 1996. Lecture Notes in Computer Science 1131, p. 75-80.

13. Schiemann, T., Tiede, U., Höhne, K. H.: Segmentation of the Visible Human for high quality volume based visualization. Med. Image Anal. 1997; 1(4):263-271.

14. Schubert, R., Höhne, K. H., Pommert, A., Riemer, M., Schiemann, T., Tiede, U.: Spatial knowledge representation for visualization of human anatomy and function. In: Barrett, H. H., Gmitro, A. F., editors. IPMI 93. Proceedings of the $13^{\text {th }}$ Conference on Information Processing in Medical Imaging; 1993 Jun 14-18; Flagstaff, Arizona. Berlin Heidelberg: Springer-Verlag 1993. Lecture Notes in Computer Science 687, p. 168-181.

15. Pommert, A., Schubert, R., Riemer, M., Schiemann, T., Tiede, U., Höhne, K. H.: Symbolic modeling of human anatomy for visualization and simulation. In: Robb R, 
editor. VBC 94. Proceedings of the Conference on Visualization in Biomedical Computing; 1994 Oct 4-7, Rochester, MN. Bellingham Washington 1994: SPIE Proceedings 2359. p. 412-423.

16. Tiede, U., Schiemann, T., Höhne, K. H.: High quality rendering of attributed volume data. In: Ebert D, et al., editors. Vis 98. Proceedings of the Conference IEEE Visualization; 1998 Oct 18-23; Research Triangle Park, NC. Los Alamitos, CA: IEEE Computer Society Press; 1998. p. 255-262.

17. Schubert, R., Pflesser, B., Pommert, A., Priesmeyer, K., Riemer, M., Schiemann, T., et al. Interactive volume visualization using "intelligent movies". In: Westwood JD et al., editors. MMVR 99. Proceedings of the Confenrence Medicine Meets Virtual Reality; 1999 Jan 20-23; San Francisco, CA. Amsterdam: IOS Press 1999. Studies in Health Technology and Informatics 62. p. 321-327.

18. Höhne, K. H., Pflesser, B., Pommert, A., Priesmeyer, K., Riemer, M., Schiemann, T., Schubert, R., Tiede, R., Frederking, H.-C., Gehrmann, S., Noster, S., Schumacher, U.: VOXEL-MAN 3D-Navigator: Inner Organs. Regional, Systemic and Radiological Anatomy. Springer-Verlag Electronic Media, Heidelberg, 2000. (3 CD-ROMs, ISBN 3540-14759-4

19. Mazziotta, J. C., Toga, A. W., Evans, A., Fox, P., Lancaster, J.: A probabilistic atlas of the human brain: theory and rationale for its development. The International Consortium for Brain Mapping (ICBM). Neuroimage.1995; 2(2): 89-101

20. Sachse, F. B., Werner, C. D., Meyer-Waarden, K., Dössel, O.: Applications of the Visible Human Male Data Set in Electrocardiology: Calculation and Visualization of Body Surface Potential Maps of a Complete Heart Cycle. In Proceedings of the $2^{\text {nd }}$ Users Conference of the National Library of Medicine's Visible Human Project, Bethesda, Maryland 1998 p. 47-48.

21. Cotin, S., Delinguette, H., Ayache, N.: Real-time elastic transformations of soft tissues for surgery simulation. IEEE Trans. Visualization Comp. Graph. 1999. 5: 62-73.

\section{Acknowledgements}

We thank Victor Spitzer and David Whitlock (University of Colorado) and the National Library of Medicine for providing the Visible Human data set. We are also grateful to Jochen Dormeier, Jan Freudenberg, Sebastian Gehrmann, and Stefan Noster for substantially contributing to the segmentation work. The knowledge modeling work was supported by the German Research Council (DFG) grant \# Ho 899/4-1. 